\title{
Toward laboratory blood test- comparable photometric assessments for anemia in veterinary hematology
}

\author{
Taehoon Kim \\ Seung Ho Choi \\ Nathan Lambert-Cheatham \\ Zhengbin $\mathrm{Xu}$ \\ Janice E. Kritchevsky \\ Francois-René Bertin \\ Young L. Kim
}




\title{
Toward laboratory blood test-comparable photometric assessments for anemia in veterinary hematology
}

\author{
Taehoon Kim, ${ }^{\text {a }}$ Seung Ho Choi, ${ }^{a}$ Nathan Lambert-Cheatham, ${ }^{a}$ Zhengbin $\mathrm{Xu},{ }^{a}$ Janice E. Kritchevsky, \\ Francois-René Bertin, ${ }^{c}$ and Young L. Kim ${ }^{\text {a,d,* }}$ \\ aPurdue University, Weldon School of Biomedical Engineering, 206 South Martin Jischke Drive, West Lafayette, Indiana 47907, United States \\ ${ }^{\text {bP }}$ urdue University, Department of Veterinary Clinical Sciences, 625 Harrison Street, West Lafayette, Indiana 47907, United States \\ 'University of Queensland, School of Veterinary Science, Outer Ring Road, UQ Gatton Campus, Gatton QLD 4343, Australia \\ ${ }^{d}$ Kyung Hee University, Department of Computer Science and Engineering, 1732 Deogyeong-daero, Yongin 7104, Republic of Korea
}

\begin{abstract}
Anemia associated with intestinal parasites and malnutrition is the leading cause of morbidity and mortality in small ruminants worldwide. Qualitative scoring of conjunctival redness has been developed so that farmers can gauge anemia in sheep and goats to identify animals that require treatment. For clinically relevant anemia diagnosis, complete blood count-comparable quantitative methods often rely on complicated and expensive optical instruments, requiring detailed spectral information of hemoglobin. We report experimental and numerical results for simple, yet reliable, noninvasive hemoglobin detection that can be correlated with laboratory-based blood hemoglobin testing for anemia diagnosis. In our pilot animal study using calves, we exploit the third eyelid (i.e., palpebral conjunctiva) as an effective sensing site. To further test spectrometerfree (or spectrometerless) hemoglobin assessments, we implement full spectral reconstruction from RGB data and partial least square regression. The unique combination of RGB-based spectral reconstruction and partial least square regression could potentially offer uncomplicated instrumentation and avoid the use of a spectrometer, which is vital for realizing a compact and inexpensive hematology device for quantitative anemia detection in the farm field. ๑ 2016 Society of Photo-Optical Instrumentation Engineers (SPIE) [DOI: 10.1117/1.JBO.21.10.107001]
\end{abstract}

Keywords: spectrometer-free; anemia; hemoglobin; noninvasive detection; partial least square regression.

Paper 160344RR received May 27, 2016; accepted for publication Sep. 14, 2016; published online Oct. 5, 2016.

\section{Introduction}

Anemia, which is defined as an absolute decrease in red blood cells in the serum, is a common heath problem in farm animals. While anemia in livestock may be associated with nutritional deficiency (e.g., iron, copper, and vitamin E), anemia in domestic animals is primarily caused by infestation by nematode parasites. $^{1,2}$ The most common test for anemia diagnosis in livestock is blood hemoglobin (Hgb) content, which is typically included in a complete blood count (CBC). Laboratory testing can be used to screen for underlying diseases before clinical signs or loss of production are present. Owing to the recent advances in hematology technologies, compact and portable (in-house) blood analyzers are currently available in clinical veterinary settings. ${ }^{3}$ However, such systems require expensive analytical cartridges with short shelf lives that only operate reliably at a constant temperature or refrigerated storage. As a result, only veterinary research centers and hospitals are able to purchase, operate, and maintain these systems. In this respect, the conjunctiva mucosa color has been assessed so that farmers can gauge anemia to identify animals that require treatments in a relatively simple manner, as shown in Fig. 1(a). The most commonly used method for conjunctival redness is the FAMACHA system, ${ }^{4,5-7}$ which allows simple clinical evaluation of anemia by use of an eye color chart. ${ }^{89}$ As a qualitative assessment of anemia, FAMACHA has been successfully used for aiding anemia diagnosis for small ruminant management,

\footnotetext{
*Address all correspondence to: Young L. Kim, E-mail: youngkim @ purdue.edu
}

particularly in sheep and goats. Although it has been tested for assessing anemia in cattle and camelids, ${ }^{5,10,11}$ FAMACHA charts have not been validated in those species. Overall, due to the limited sensitivity and specificity, this would be inappropriate for being used on its own as a stand-alone system.

In search for noninvasive anemia detection strategies with high accuracy and sensitivity comparable to blood Hgb concentration (i.e., gold standard and definitive diagnosis), it appears that the eye conjunctiva would be an ideal anemia detection site due to the microvasculature exposed on the mucosal surface. ${ }^{12,13}$ Indeed, optical assessment of the inner eyelid has received attention in anemic patients for assessing Hgb content from human individuals, which could be highly correlated with a lab-based blood Hgb test. ${ }^{14-19}$ For accurate and precise Hgb measurements, typical spectroscopic technologies heavily rely on the use of spectrometers, spectrographs, and liquid tunable crystal filters. ${ }^{20,21}$ However, these critical optical components for highly accurate and sensitive $\mathrm{Hgb}$ measurements significantly limit the development of compact, portable, and cost-effective devices. For example, such spectroscopic measurements would not be ideal for developing compact and economical photometric anemia detection that could potentially be integrated into smartphones. In addition, although there is a variety of mobile $\mathrm{Hgb}$ measurement devices for humans, they have not been evaluated for clinical use in other species.

In this study, we demonstrate the feasibility of simple, yet reliable, noninvasive $\mathrm{Hgb}$ detection that can be significantly 

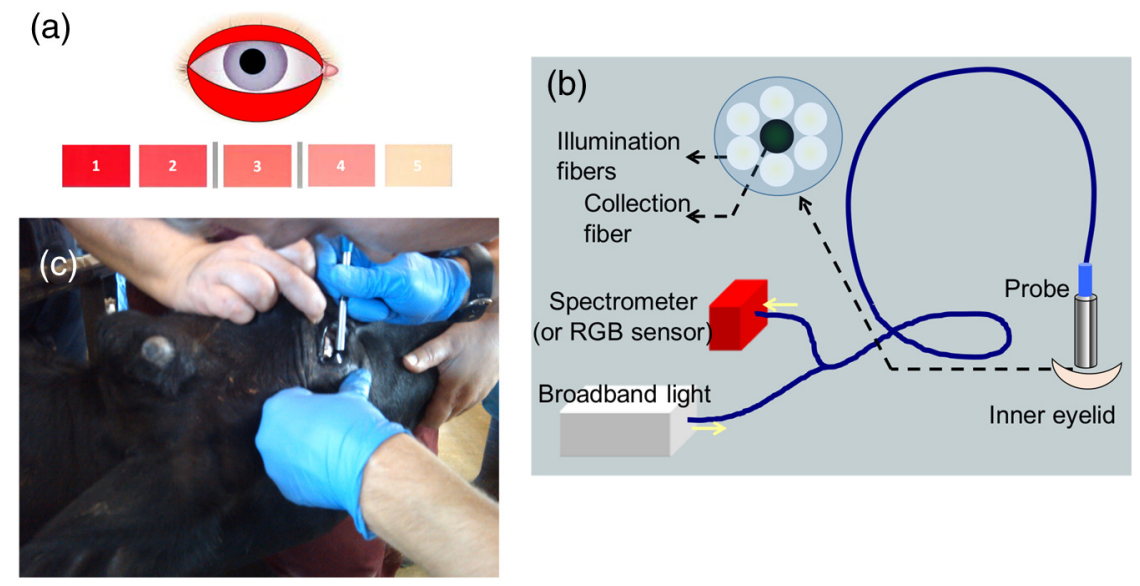

Fig. 1 (a) Qualitative scoring of the conjunctiva mucosa color so that farmers can gauge anemia to identify animals that require treatments. (b) Probe configuration and system setup for optical reflectance measurements. (c) Photography of data acquisition from the third eyelid of a calf in the farm field.

correlated with laboratory blood Hgb test for anemia diagnosis in livestock. The main objective is to test optical Hgb measurement methods that can be highly comparable to blood Hgb tests in CBC. We report experimental and numerical results from a pilot animal study in cattle, in particular calves. As an effective sensing site, we exploit the third eyelid (i.e., palpebral conjunctiva) of cattle that have easily accessible microvasculature, because other absorbers (e.g., melanin, bilirubin, and $\beta$-carotene) in the skin can be fundamental obstacles for accurately assessing $\mathrm{Hgb}$ content for reliable anemia detection. For a simple detection system, we make use of an optical reflectance probe consisting of illumination and collection channels coupled with a visible light source and a spectrometer, respectively. A relatively large contacting area of the optical probe minimizes potential measurement variations associated pressure exerted on the tissue surface. Applying partial least square regression (PLSR) for spectral correlations with blood Hgb content, we analyze spectral data obtained from a series of phantom studies as well as bull calves from a commercial veal calf facility. To further test the concept of spectrometerless (or spectrometer-free) methods, we numerically convert full spectral information to RGB signals that could easily be acquired using a three-color sensor camera. By inversely reconstructing the full spectra from the RGB signals, we compare correlations between eyelid $\mathrm{Hgb}$ and conventional blood $\mathrm{Hgb}$ content in CBC. We envision that the proposed detection scheme will offer simple instrumentation and avoid the use of a spectrometer, which will be vital for realizing an uncomplicated and costeffective hematology device for quantitative anemia detection in the farm field.

\section{Methods and Algorithms}

\subsection{Blood Hgb Correlation Models Using Full Spectra Data}

To identify correlations between measured reflectance spectra and blood Hgb (i.e., gold standard and definitive diagnosis), we made use of a PLSR method. PLSR has been broadly used to model relationships among observed variables and response (or outcome) variables in the fields of chemometrics, food safety, industrial processes, and system biology. ${ }^{22-28}$ Because PLSR assumes that observed variables (i.e., predictors) for characterizing a system or a process can be represented by a small number of latent variables (also known as components), it can work effectively, in particular, for analyzing a wide spectrum of biological data. Inherently, reflectance spectra (i.e., observed variables) often have multicollinearity as hyperspectral information. Thus, only a few underlying latent variables, which are linear combinations of original observed variables, are likely to represent most of variations in the original predictors. Although PLSR is similar to principal component regression (PCR) in terms of extracting principal components, it clearly distinguishes itself from PCR by taking variations in predictors and response variables (i.e., outcome variables) into account simultaneously. ${ }^{29,30}$ PLSR projects high-dimensional original variables (e.g., reflectance spectra) onto a lower space of latent variables. Such transformation allows us to examine the significance of individual observed variables, making it possible to eliminate insignificant variables as variable selection methods. ${ }^{31}$ Overall, PLSR is a distinct method for constructing a predictive model by including both predictor variables and response variables when the predictor variables are highly collinear.

In addition, the use of PLSR is beneficial in avoiding overfitting when the size of predictors is larger than the sample size, in which multivariate linear regression is often prone to overfitting. ${ }^{30}$ In our previous studies, ${ }^{32-34}$ we intensively used model-based $\mathrm{Hgb}$ detections that require a priori information of the absorption spectra of all possible absorbing molecules in the skin. However, in this method, all possible absorbers in tissue should be included for highly reliable Hgb quantification, which is the primary objective of this study. In this respect, PLSR provides an alternative yet efficient method for Hgb spectral analyses by incorporating other potential yet minimal spectral features for accurate and sensitive Hgb quantification. Given such suitability for spectral analyses, we applied PLSR to build a model to determine blood Hgb levels (i.e., outcome variable) from measured reflectance spectra (i.e., predictors) in our study. After the model was trained appropriately, we further evaluated the ability for predicting blood Hgb levels for new spectral reflectance data using 10 -fold cross-validation.

\subsection{Full Spectral Reconstruction from RGB}

Recent optical sensing technologies heavily rely on spectrometers, spectrographs, and tunable color filters, all of which 
significantly limit the development of simple and economical devices. ${ }^{21,35,36}$ As the first step for realizing blood Hgb testcomparable photometric anemia detection superior to the conventional color chart methods (e.g., FAMACHA), we investigated spectral reconstruction from RGB data that are commonly acquired from conventional cameras. This approach of spectral reconstruction from RGB data could potentially offer simple instrumentation and operation, using the currently available smartphone technologies. ${ }^{21,35,36}$ In this respect, we performed numerical experiments to test the feasibility that a blood $\mathrm{Hgb}$ level can be sensitively and accurately predicted using a three-color sensor-based system in a similar manner of our previous study. ${ }^{21}$ First, RGB signals were acquired from measured reflectance spectra data. A camera response of RGB signals were computed such that

$x_{3 \times 1}=S_{3 \times N} r_{N \times 1}+e_{3 \times 1}$,

where $x$ is a $3 \times 1$ vector corresponding to RGB signals, $S$ is a $3 \times N$ matrix of the spectral sensitivities of the RGB camera sensor, $r$ is an $N \times 1$ vector that has the reflectance intensity as a function of wavelength $\lambda$, and $e$ is a $3 \times 1$ vector of system noise with zero mean. $N$ indicates the number of wavelengths. In our case, $N=201$ from 400 to $800 \mathrm{~nm}$ with a spectral interval of $2 \mathrm{~nm}$. In general, $S$ can be obtained from the sensor manufacturer or be estimated mathematically from training color samples. ${ }^{37,38}$ In our case, the spectral sensitivities of a threecolor CCD sensor (Sony ICX625) were used to obtain RGB signals from the measured reflectance spectra,

$\hat{R}_{m \times N}=X_{m \times 3} T_{3 \times N}$.

Second, once RGB signal $x_{3 \times 1}$ was obtained for each spectrum, $\hat{R}_{m \times N}$ and $X_{m \times 3}$ were constructed by stacking the reflectance spectra $r_{N \times 1}$ and RGB data $x_{3 \times 1}$ from several independent samples, where $m$ is the number of different samples and $N$ is the number of wavelengths. $\hat{R}_{m \times N}$ and $X_{m \times 3}$ are the collections of the measured reflectance spectra and RGB data that are built up from $m$ different samples. With this data set, we learned a transformation matrix $T_{3 \times N}$ that converts RGB data into reflectance spectra, which is unknown and can be solved from Eq. (2). Then, we can predict a reflectance spectrum $\hat{r}$ from a new RGB signal $x$ by multiplying $x$ by $T$. To improve the prediction accuracy for spectral reconstruction, we selected multivariate second-order polynomial regression with leave-one-out crossvalidation. $^{21,35}$ Third, using the reconstructed reflectance spectra, we reliably quantified blood $\mathrm{Hgb}$ levels using the PLSR method described in Sec. 2.1. In this final step, we used 10-fold cross-validation to strengthen the validation process of the Hgb correlation model in which a testing data set did not include any training data sets. Given the bovine data set, we used the in-sample data for building the correlation model and employed the out-of-sample data to test the performance of the model. Thus, we were able to avoid overfitting of the data by not reusing the same data to both train and test the model.

\section{Materials and Experiments}

\subsection{Experimental Setup}

To acquire reflectance spectra on the third eyelid of calves, we used a commercially available fiber-optic-based probe (Ocean Optics, Inc., Dunedin, Florida) with an outer diameter

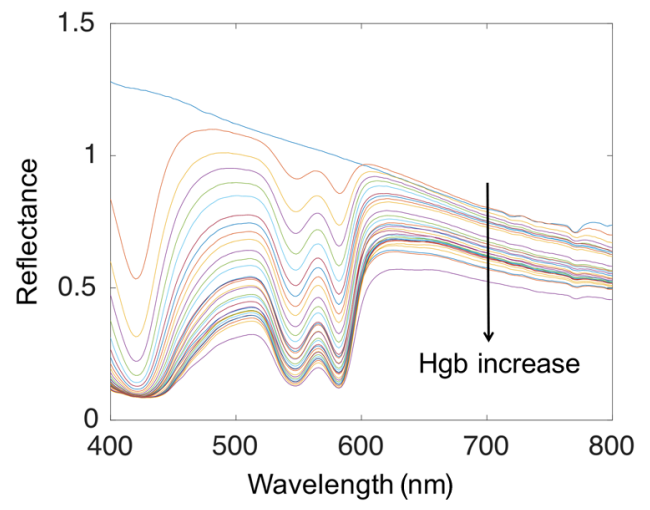

Fig. 2 Representative spectra of various $\mathrm{Hgb}$ content from a series of tissue phantoms. The overall reflectance intensity for each Hgb sample decreases as Hgb content increases, while the unique Hgb spectral signatures at the Soret and $\mathrm{Q}$ bands become stronger. The top spectrum does not include any $\mathrm{Hgb}$.

of $6.35 \mathrm{~mm}(1 / 4 \mathrm{in}.) .{ }^{39,40}$ Our intention of using the relative large-diameter optical probe was to minimize spectral variations associated the pressure exerted from the probe in contact with the tissue surface. ${ }^{41-45}$ In particular, the effect of probe contact pressure is known to be significant with small-diameter miniature probes due to mechanical indentation effects. The optical reflectance probe consisted of one central optical fiber and six surrounding fibers, which work as a collection port and illumination ports, respectively. All of the fibers had the core dimeter of $400 \mu \mathrm{m}$ with a numerical aperture of 0.22 (i.e., acceptance angle of $\sim 25 \mathrm{deg}$ in air), and the separation distance between the collection and illumination was $\sim 0.5 \mathrm{~mm}$. A tungsten-halogen lamp (Ocean Optics, Inc., Dunedin, Florida) was coupled to the illumination ports, and a spectrometer in a range of 400 to $800 \mathrm{~nm}$ with a spectral resolution of $2 \mathrm{~nm}$ (Horiba Jobin Yvon, Edison, New Jersey) was coupled to the collection port. We also removed the background light and compensated for the system spectral responses using a reflectance reference standard (Labsphere, North Sutton, New Hampshire). ${ }^{21}$ Figure 1(b) shows the optical system configuration for reflectance spectra data acquisition in a farm field, as shown in Fig. 1(c).

\subsection{Tissue Phantom Test}

To evaluate PLSR and the spectral reconstruction method under well-controlled conditions, we conducted a series of tissue phantom studies consisting of aqueous suspensions of microspheres (i.e., scatterers) and solutions of lyophilized Hgb (i.e., absorbers) in a similar manner in our previous studies. ${ }^{32,34}$ We first estimated the scattering properties (i.e., transport mean free path length and anisotropy factor) of the scattering media using Mie theory. ${ }^{46}$ Polystyrene microspheres with a nominal diameter of $0.36 \mu \mathrm{m}$ (Thermo Fisher Scientific Inc., Waltham, Massachusetts) were used, resulting in a scattering pathlength of $90 \mu \mathrm{m}$ and a anisotropy factor of 0.73 at $\lambda=600 \mathrm{~nm}$. Second, we gradually increased $\mathrm{Hgb}$ content by adding lyophilized $\mathrm{Hgb}$ (Sigma-Aldrich, St. Louis, Missouri) into the scattering suspension from 0 to $\sim 23 \mathrm{mg} / \mathrm{mL}$. The optical probe was submerged in the suspension with a total volume of $21.5 \mathrm{~mL}$ and a height of $15.0 \mathrm{~mm}$ and reflectance spectra were recorded. Figure 2 shows representative spectra from the tissue phantoms as a function of $\mathrm{Hgb}$ content. The overall reflectance intensity for each $\mathrm{Hgb}$ sample decreases as Hgb content increases, while the unique 

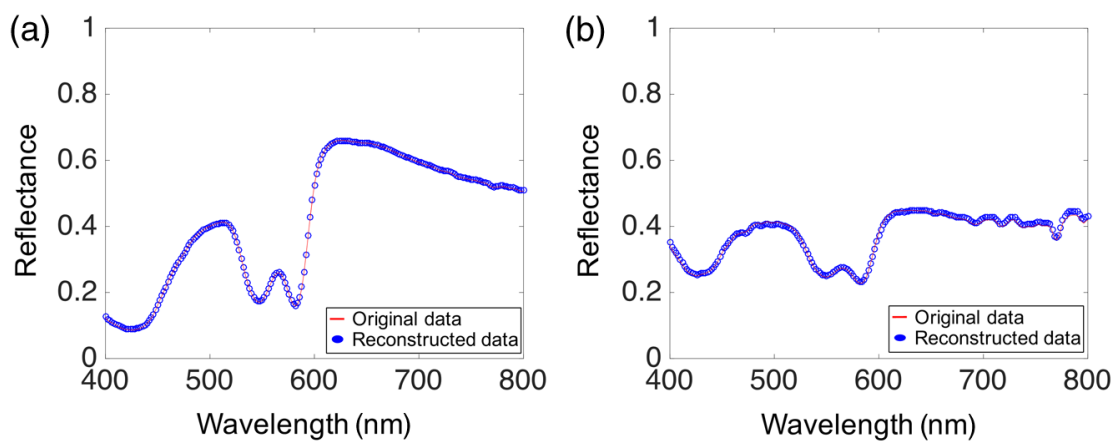

Fig. 3 (a, b) Spectra reconstructed from RGB signals in representative phantom and bovine samples, respectively. The reconstructed spectra significantly resemble the original spectra.

$\mathrm{Hgb}$ spectral signatures around 420, 540, 560, and $580 \mathrm{~nm}$ in the visible wavelength range (i.e., Soret and $\mathrm{Q}$ bands) are manifested.

\subsection{Pilot Animal Study: Blood Hgb Testing and Conjunctival Redness Scoring}

To evaluate the feasibility of blood Hgb test-comparable optical $\mathrm{Hgb}$ assessments in animals, we tested a total of 56 Holstein bull calves at $\sim 4$ months of age that were located in a commercial veal calf facility in Ohio. They were pen-housed in groups of three from the time they arrived at $\sim 4$ to 6 weeks of age until they were marketed at 6 months of age. During the growing process, blood was routinely collected periodically from either all the calves or a subset of calves for CBC determination. The producers used blood $\mathrm{Hgb}$ values to determine whether any calves require iron supplementation. For our study, immediately before or after optical spectral measurements, we collected blood with a volume of $5 \mathrm{~mL}$ from the jugular vein into an evacuated ethylenediamine tetraacetic acid tube as an anticoagulant for blood collection. We made spectral measurements by lightly placing the optical probe against the third eyelid of restrained calves. We analyzed blood Hgb using a commercially available hematology system (CELL-DYN 3700, Abbott Diagnostics, Lake Forest, Illinois). Primarily, as the gold standard for definitive diagnosis, blood $\mathrm{Hgb}$ levels were used to correlate with spectral reflectance readings from the eyelid. For a subset of calves $(n=31)$, we also obtained FAMACHA-like conjunctival redness readings in a score of 1 to 5 [pink/red to white mucous membranes in Fig. 1(a)] from two trained veterinarians and selected the lower score between two independent scores in each animal.

\section{Results}

\subsection{Reconstruction of Reflectance Spectra from $R G B$}

As shown in Figs. 3(a) and 3(b), the representative full spectra reconstructed from the RGB signals significantly resemble the original spectra in both phantom and bovine samples. Specifically, we estimated prediction errors between the reconstructed reflectance spectra and the original spectra. When $95 \%$ confidence intervals of mean differences (dark area) at each wavelength are shown in Fig. 4, the reconstruction error patterns (confidence intervals) in wavelength are strongly associated with the spectral sensitivities of the RGB camera sensor but not much related to the samples. For both phantom and bovine experiments, the confidence intervals at around $\lambda=400 \mathrm{~nm}$ and $\lambda=700 \mathrm{~nm}$ become wider. These limitations correspond directly to the low spectral sensitivities of the RGB camera as included on the background of Fig. 4. We also validated the reconstruction algorithm using leave-one-out cross-validation due to the relative small sample size. For the phantom study and the bovine experiment, the average $R^{2}$ were 0.999 and 0.985 , and the average RMSE of cross-validation were 0.0024 and 0.0068 , respectively. As expected, the phantom samples in the strictly controlled environment have enhanced
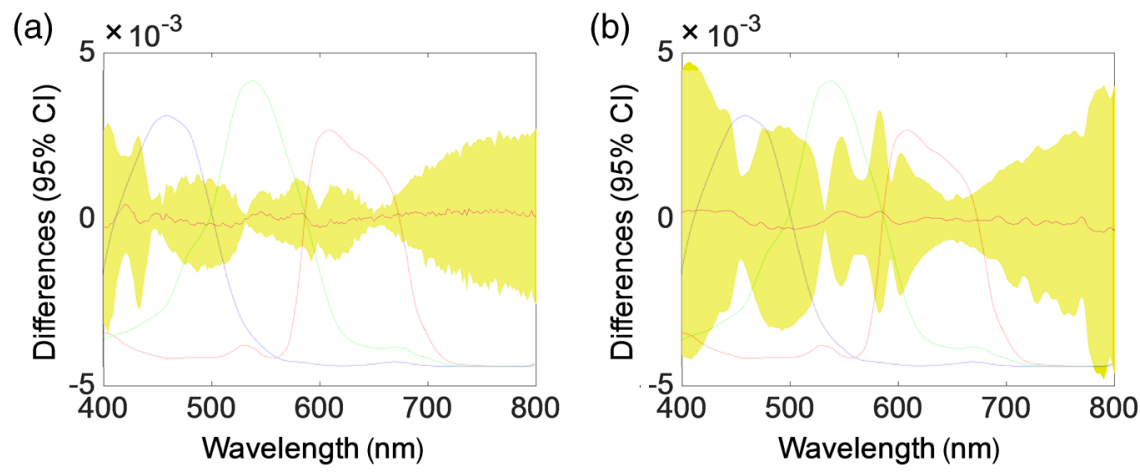

Fig. 4. (a, b) Estimated prediction errors between reconstructed reflectance spectra and original spectra in phantom and bovine samples, respectively. $95 \%$ confidence intervals of mean differences (dark area) at each wavelength are depicted. The confidence intervals at around $\lambda=400 \mathrm{~nm}$ and $\lambda=700 \mathrm{~nm}$ become wider. These limitations are related to the low spectral sensitivities of the RGB camera as shown on the background (blue, green, and red curves). 

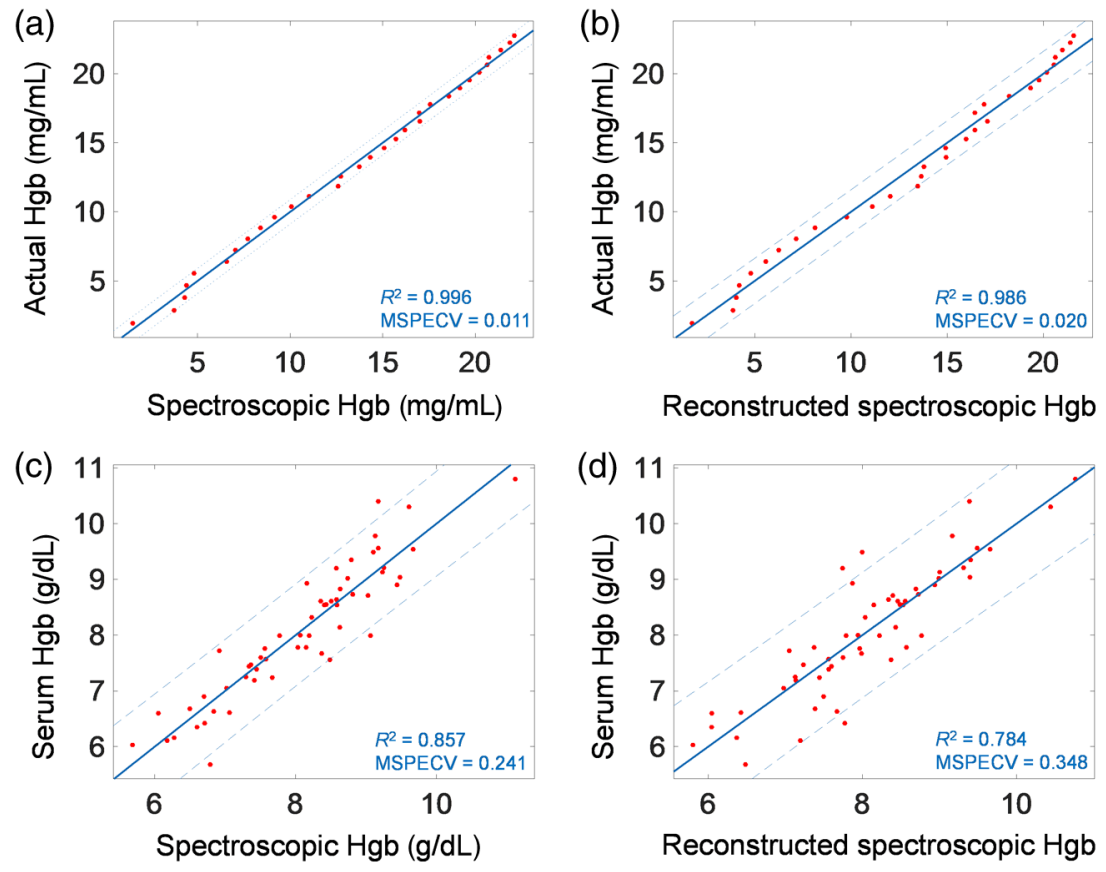

Fig. $5(a, b)$ Correlations between actual and optically measured $\mathrm{Hgb}$ values from the full spectral data and the RGB data in the phantom study, respectively. Due to the reconstruction from RGB, the $R^{2}$ value in (b) is slightly lower than that of the full spectral data in (a). (c, d) Correlations between actual and optically measured $\mathrm{Hgb}$ values from the full spectral data and the RGB data in the bovine study, respectively. Compared with those of the phantom study, the correlation values for both original and reconstructed reflectance spectra are slightly reduced, due to several factors including a narrow range of blood Hgb levels in our study population.

accuracy for the spectral reconstruction than the bovine ones. The bovine result still supports the feasibility of the RGBbased reconstruction approach that a blood Hgb level can be accurately predicted using a three-color sensor-based system.

\subsection{Blood Hgb Correlations with RGB}

Based on the full reflectance spectra reliably reconstructed from the RGB signals above, we determined correlations between actual $\mathrm{Hgb}$ and estimated $\mathrm{Hgb}$ from the reflectance data, using PLSR. For the phantom study, the PLSR method reliably predicted actual $\mathrm{Hgb}$ using the reflectance spectra or the RGB data. Figures 5(a) and 5(b) show correlations between the actual and optically measured $\mathrm{Hgb}$ values from the full spectral data and the RGB data in the phantom study, respectively. Obviously, contributing from the reconstruction error from RGB, the $R^{2}$ value in Fig. 5(b) is slightly lower than that of the full spectral data. For the bovine study, the $R^{2}$ value of 0.857 shows the feasibility of the blood $\mathrm{Hgb}$ comparability using the full spectral reflectance measurements [Fig. 5(c)]. On the other hand, the correlations for both original and reconstructed reflectance spectra are reduced as shown in Figs. 5(c) and $5(\mathrm{~d})$.

We also report mean squared prediction errors (MSPECV) through 10-fold cross-validation for each case in Fig. 5, which is an important metric of prediction accuracy for the PLSR models. Figures 6(a) and 6(b) show changes in MSPECV as the number of components increases in the PLSR model.
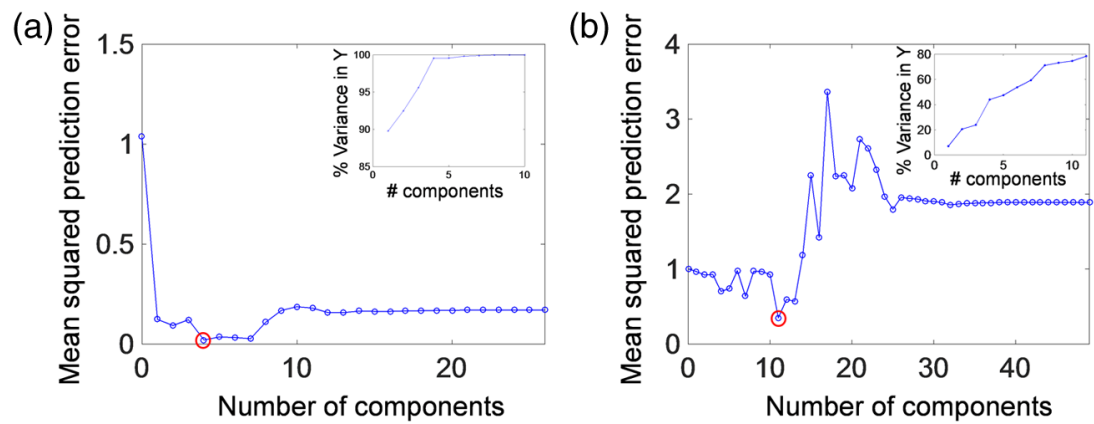

Fig. 6 (a, b) Changes in MSPECV as a function of the number of PLS components in the phantom and bovine studies, respectively. Although an increased number of components in the PLSR model results in low prediction errors, MSPECV reaches to optimal numbers of PLS components [i.e., four in (a) and 11 in (b)]. Insets: The cumulative percentage of variances in the response variable (i.e., true $\mathrm{Hgb}$ ) as a function of the number of components. 
Initially, an increased number of components in the PLSR model contributes to better representation of variations in the observed variables (i.e., reflectance spectra) and the response variables (i.e., lab-based blood $\mathrm{Hgb}$ ) simultaneously, thus making its prediction errors lower. As shown in the insets of Fig. 6, each component contributes to different percentage variations accounted for the response variables. However, as the number of partial least square (PLS) components increases, MSPECV reaches an optimal number of PLS components. Above these levels, the PLSR model starts overfitting, meaning that random noises in the spectral data can be involved in the model. As a result, the prediction error mostly increases again. In this aspect, we note that $R^{2}$ would not be an ideal metric to use for assessing its predictive ability because $R^{2}$ continues to grow with an increase in the number of components.

In the PLSR model, the degree of freedom can be understood in term of the number of components, which also captures the complexity of the model and is useful for determining the final model. Basically, our Hgb correlation model using PLSR allows us to increase the degree of freedom to have a higher correlation of optical Hgb and actual Hgb. However, we cannot continue to add more components as the complexity of the model increases. Indeed, for our final models, we limited the number of components, because a higher degree of freedom adds more errors. The optimal numbers of PLS components were determined to be four and 11 in the phantom and bovine studies, respectively. The behavior of the phantom data can be explained by a simpler model, compared with the bovine data.

\section{Discussion}

\subsection{Limitations of Conjunctival Redness Reading}

The presented method could potentially be useful for livestock that cannot be evaluated with the FAMACHA system. While FAMACHA is well established for sheep and goats, it has not yet been validated for cattle and calves. ${ }^{5,10,11}$ Such a conjunctival redness scoring system has limited applications in cattle and calves, although some studies found that hematocrit is linearly correlated with FAMACHA scores in calves. ${ }^{47}$ Indeed, we found no evidence of associations of conjunctival redness scores with blood Hgb levels in our study population. The conjunctival redness scoring result from a subset of the calves shows no significant correlation with blood $\mathrm{Hgb}$ levels $\left(R^{2}=0.004\right.$ with a $p$-value of 0.74$)$, as shown in Fig. 7(a).
This null result of the FAMACH-like test indicates that conjunctival redness scoring in calves may be presented in a delayed manner for clinical anemia, compared with sheep and goats. Thus, our proposed method could potentially be beneficial for anemia detection of calves and cattle. The fact that the probe was physically placed on the third eyelid required the calf to be restrained for a short period of time but was less invasive than collecting blood.

\subsection{Limitations of Conventional RGB-Based Photometric Technologies}

Clinical examination of pallor in conjunctiva, nailbed, tongue, and palm has been extensively studied for cost-effective and simple anemia assessments in humans. ${ }^{16,19,48}$ Although conjunctival pallor is valuable information as a clinical sign for severe anemia, it cannot provide reliable diagnosis in clinical settings. Further, quantification of $\mathrm{Hgb}$ from conjunctival pallor using digital photography has received attention. ${ }^{17,18}$ The common agreement in the medical community is that this method still lacks sensitivity and specificity for anemia diagnosis in conventional clinical settings. As a result, these previous studies strongly indicate that RGB information would not be sufficient to have reliable correlations with actual Hgb content. In our study, we also conducted multiple linear regression of blood $\mathrm{Hgb}$ levels (i.e., outcome variable) against RGB (i.e., predictor variables). Although the $\mathrm{R}$ (red) channel is associated with blood Hgb with a $p$-value $=0.047$, the correlation coefficient is extremely low with $R^{2}=0.26$ in Fig. 7(b). This regression result clearly supports the idea that detailed spectral information is mandatory for computing an absolute value of $\mathrm{Hgb}$ content. We note that this limitation of mere RGB information is also reflected in the conjunctival redness scoring result reported in Sec. 5.1. [Fig. 7(a)]. In this respect, the mathematical reconstruction of hyperspectral data from RGB can offer a new class of conjunctival pallor assessments for laboratory blood test-comparable anemia diagnosis.

\subsection{Partial Least Square Component Analyses}

To interpret the characteristics of the PLS components, we conducted spectral analyses of PLSR for the phantom study and bovine experiments. In Fig. 8, PLS components depict how the original variables (i.e., spectral reflectance intensities at each wavelength) contribute to the formation of the PLSR
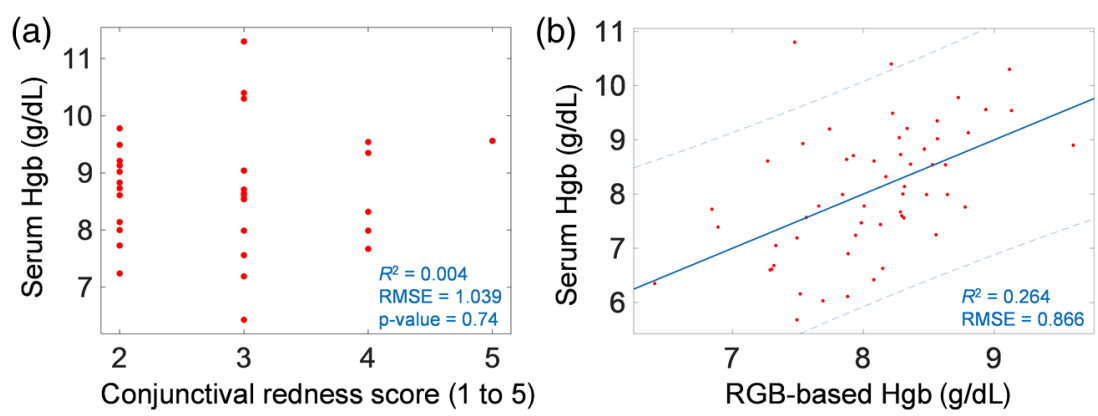

Fig. 7 (a) Association of conjunctival redness with blood Hgb levels. The conjunctival redness scoring from a subset of the calves $(n=31)$ shows no significant correlation with blood Hgb levels, supporting the idea of RGB camera-based photonumeric anemia detection. (b) Blood Hgb correlation with mere RGB without spectral reconstruction in the bovine study. The result of multiple linear regression shows that mere RGB data without reconstruction do not provide sufficient information to reliably assess blood $\mathrm{Hgb}$ test-comparable Hgb. 

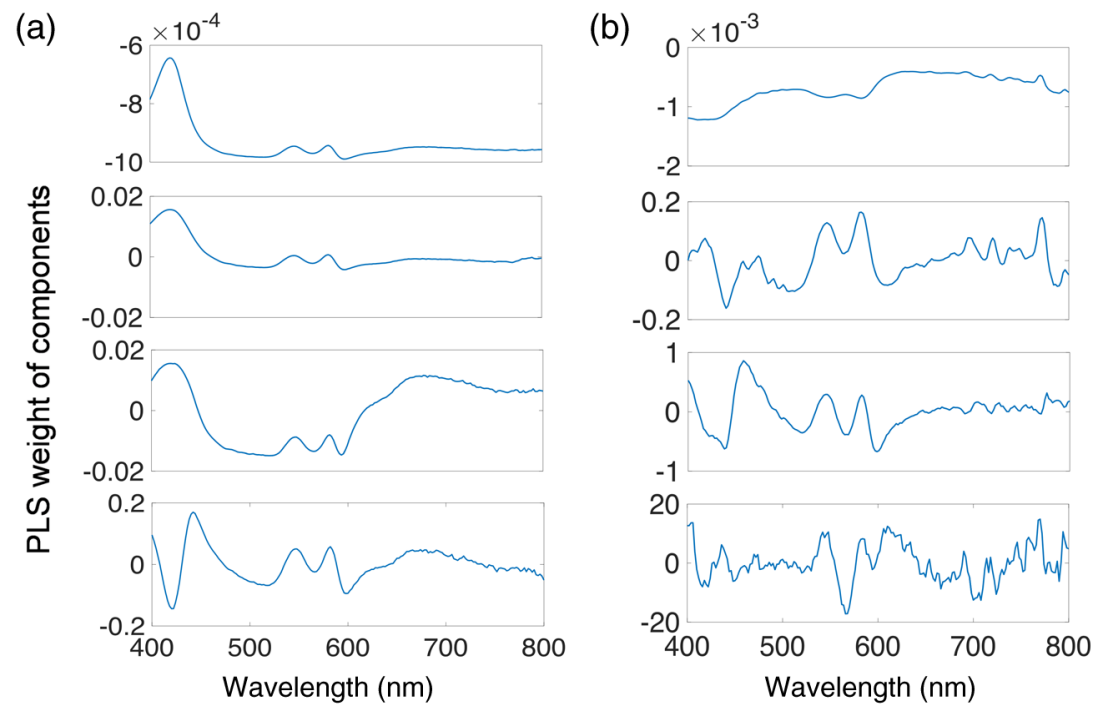

Fig. 8 (a, b) PLS weights of representative PLS components in the phantom and bovine spectral analyses, respectively. For the phantom data, first to fourth components are selected and for the bovine data, 1st, 5th, 7th, and 11th components are selected for display. The PLS weights show how strongly each component depends on the original predictors.

model. The PLS weights of components show which predicator variables are associated with the response variable and which ones convey critical information for identifying correlations with actual Hgb and spectroscopic Hgb. Several components have the unique $\mathrm{Hgb}$ spectral pattern around 420, 540, 560, and $580 \mathrm{~nm}$ in both phantom and bovine studies, which correspond to Soret and Q bands of Hgb molecules. Compared to the phantom study [Fig. 8(a)], the PLS weights of components in the bovine study are noisy especially above $650 \mathrm{~nm}$. However, the similar spectral peaks or dips around 420, 540, 560 , and $580 \mathrm{~nm}$ still reflect the $\mathrm{Hgb}$ absorption signatures in the visible range. Overall, the spectral shapes of the components are in good agreement with the Hgb signatures shown in the reflectance spectra (Figs. 2 and 3).

\subsection{Probe Contact Pressure Issues}

Although the relatively large-area probe was employed in contact with the third eyelid of calves, the probe pressure effect cannot be ruled out in our animal study, given that the immobility of the animals were not ensured during optical readings. Indeed, the influence of probe contact pressure on spectral reflectance measurements of tissue has been intensively investigated, because the exerted pressure can reduce local blood volumes. ${ }^{42-45}$ Spectral alterations and temporal variations associated with contact pressure are a critical challenge for optical diagnosis in conjunction with endoscopy. In this respect, we are currently considering a few different noncontact configurations that can be implemented using RGB cameras or smartphones. We further expect that a camera-based FAMACH-like system will allow clinically relevant assessments of anemia, highly comparable to lab-based blood Hgb tests in cases where conjunctival redness readings are challenging.

\subsection{Limitations of Our Study}

Although other studies have examined the relationship between anemia and RGB photometric information, to our knowledge this is the first to examine associations between true $\mathrm{Hgb}$ and reconstructed full spectra in the third eyelid of calves. However, our animal study has limitations: first, a potential pitfall for the proposed training-based approach, such as RGBbased reconstruction, and PLSR is the requirement of a wide range of training set of reflectance spectra for accurate and precise prediction of actual $\mathrm{Hgb}$. As a universal limitation for any training-based approaches, this requirement can involve a proper selection of samples, which in turn affects the prediction performance of estimating true blood Hgb levels. As discussed in Sec. 4.2. Blood Hgb correlations with RGB, one possible source of the lower performance in the bovine study stems from the narrow range of blood Hgb levels only from 6 to $11 \mathrm{~g} / \mathrm{dL}$. The normal range of $\mathrm{Hgb}$ is 9 to $15 \mathrm{~g} / \mathrm{dL}$ in cattle and the cutoff for anemia is $7 \mathrm{~g} / \mathrm{dL}$ in veal calves. This means that our study population included limited cases of moderate and severe anima. In addition, the lower $R^{2}$ values in the animal study can be attributable to other several factors in the measurement environment, including the movement of calves during optical reading on the eyelid and the limited control of the ambient light in the farm field.

\section{Conclusion}

We have demonstrated the feasibility of blood Hgb test-comparable optical $\mathrm{Hgb}$ measurements at the third eyelid, which overcomes the limitation of conjunctival redness scoring in calves and cattle. In our animal study, we have incorporated optical device instrumentation, clinical hematology laboratory, and routine clinical examination in veterinary medicine. To enhance the correlation with blood Hgb levels, we have combined PLSR and full spectral reconstruction from RGB using spectral measurements on the third eyelid. Owing to the microvasculature exposed on the tissue surface, the third eyelid can be an ideal tissue site for assessing $\mathrm{Hgb}$ from individual animals. We have shown that the spectral analyses of PLSR using spectra reconstructed from RGB allows for accurately quantifying blood $\mathrm{Hgb}$ levels. We note that the current Hgb prediction model may be applicable for a relatively small range of blood Hgb levels in calves, due to the limited model training of the current 
hyperspectral reconstruction method. Systematic studies including a clinically relevant large range of blood $\mathrm{Hgb}$ levels will allow us to develop accurate and precise prediction models in different species of animals. We envision that the reconstruction of detailed spectra from RGB signals could potentially offer simple instrumentation and avoid the use of a spectrometer, which is critical to develop a compact RGB camera-based anemia detection device. After establishing a stronger correlation with blood $\mathrm{Hgb}$ levels in a noncontact manner, we further expect that a needleless, noninvasive, cost-effective, user-friendly camera adjuvant for anemia examination will play a critical role in the accurate diagnosis of anemia in resource-limited settings in both veterinary medicine and human medicine.

\section{Acknowledgments}

We acknowledge the support from Samsung Global Research Outreach Program and Faculty Fellowship Program from Air Force Research Laboratory's Materials and Manufacturing Directorate. The authors have no relevant financial interests and no other potential conflicts of interest to disclose.

\section{References}

1. M. J. Mcarthur and C. R. Reinemeyer, "Herding the US cattle industry toward a paradigm shift in parasite control," Vet. Parasitol. 204(1), 34-43 (2014).

2. D. H. Bliss, R. D. Moore, and W. G. Kvasnicka, "Parasite resistance in US cattle," Am. Assoc. Bovine Pract. 41, 109-114 (2008)

3. L. Roland, M. Drillich, and M. Iwersen, "Hematology as a diagnostic tool in bovine medicine," J. Vet. Diagn. Invest. 26(5), 592-598 (2014).

4. C. S. Sotomaior et al., "Sensitivity and specificity of the FAMACHA" system in Suffolk sheep and crossbred boer goats," Vet. Parasitol. 190(1), 114-119 (2012).

5. D. Grace et al., "Comparing FAMACHA ${ }^{\odot}$ eye color chart and hemoglobin color scale tests for detecting anemia and improving treatment of bovine trypanosomosis in West Africa," Vet. Parasitol. 147(1), 26-39 (2007).

6. R. M. Kaplan et al., "Validation of the FAMACHA ${ }^{\odot}$ eye color chart for detecting clinical anemia in sheep and goats on farms in the southern united states," Vet. Parasitol. 123(1), 105-120 (2004).

7. J. A. Van Wyk and G. F. Bath, "The FAMACHA ${ }^{\odot}$ system for managing haemonchosis in sheep and goats by clinically identifying individual animals for treatment," Vet. Res. 33(5), 509-529 (2002).

8. D. P. Reynecke et al., "Validation of the FAMACHA ${ }^{\mathcal{O}}$ eye colour chart using sensitivity/specificity analysis on two South African sheep farms," Vet. Parasitol. 177(3), 203-211 (2011).

9. D. G. Riley and J. A. Van Wyk, "Genetic parameters for FAMACHA ${ }^{\odot}$ score and related traits for host resistance/resilience and production at differing severities of worm challenge in a merino flock in South Africa," Vet. Parasitol. 164(1), 44-52 (2009).

10. P. Dorny et al., "Infections with gastrointestinal nematodes, fasciola and paramphistomum in cattle in cambodia and their association with morbidity parameters," Vet. Parasitol. 175(3-4), 293-299 (2011).

11. J. A. V. Wyk and D. P. Reynecke, "Blueprint for an automated specific decision support system for countering anthelmintic resistance in haemonchus spp. At farm level," Vet. Parasitol. 177(3-4), 212-223 (2011).

12. R. G. Nadeau and W. Groner, "The role of a new noninvasive imaging technology in the diagnosis of anemia," J. Nutr. 131(5), 1610S-1614S (2001).

13. A. R. Kent, S. H. Elsing, and R. L. Hebert, "Conjunctival vasculature in the assessment of anemia," Ophthalmology 107(2), 274-277 (2000).

14. J. Mcmurdy et al., "Photonics based in vivo total hemoglobin monitoring and clinical relevance," J. Biophotonics 2(5), 277-287 (2009).

15. J. W. Mcmurdy et al., "Diffuse reflectance spectra of the palpebral conjunctiva and its utility as a noninvasive indicator of total hemoglobin," J. Biomed. Opt. 11(1), 014019 (2006).

16. T. N. Sheth et al., "The relation of conjunctival pallor to the presence of anemia," J. Gen. Intern. Med. 12(2), 102-106 (1997).
17. S. Suner et al., "Non-invasive determination of hemoglobin by digital photography of palpebral conjunctiva," J. Emerg. Med. 33(2), 105-111 (2007).

18. S. Collings et al., "Non-invasive detection of anaemia using digital photographs of the conjunctiva," PLoS One 11(4), e0153286 (2016).

19. A. Kalantri et al., "Accuracy and reliability of pallor for detecting anaemia: a hospital-based diagnostic accuracy study," PLoS One 5(1), e8545 (2010).

20. S. J. Woltman, G. D. Jay, and G. P. Crawford, "Liquid-crystal materials find a new order in biomedical applications," Nat. Mater. 6(12), 929938 (2007).

21. T. Kim et al., "Nonspectroscopic imaging for quantitative chlorophyll sensing," J. Biomed. Opt. 21(1), 016008 (2016).

22. D. V. Nguyen and D. M. Rocke, "Tumor classification by partial least squares using microarray gene expression data,' Bioinformatics 18(1), 39-50 (2002).

23. N. J. Lobaugh, R. West, and A. R. Mcintosh, "Spatiotemporal analysis of experimental differences in event-related potential data with partial least squares," Psychophysiol. 38(3), 517-530 (2001).

24. J. Hulland and R. I. S. O. Business, "Use of partial least squares (PLS) in strategic management research: a review of four recent studies," Strategic Manage. J. 20(2), 195-204 (1999).

25. L. M. Carrascal, I. Galvan, and O. Gordo, "Partial least squares regression as an alternative to current regression methods used in ecology," Oikos 118(5), 681-690 (2009).

26. L. Cerretani et al., "Application of partial least square regression to differential scanning calorimetry data for fatty acid quantitation in olive oil," Food Chem. 127(4), 1899-1904 (2011).

27. S. A. Mahesar et al., "Determination of total trans fat content in Pakistani cereal-based foods by SB-HATR FT-IR spectroscopy coupled with partial least square regression," Food Chem. 123(4), 1289-1293 (2010).

28. H. T. Nguyen and B.-W. Lee, "Assessment of rice leaf growth and nitrogen status by hyperspectral canopy reflectance and partial least square regression," Eur. J. Agron. 24(4), 349-356 (2006).

29. H. Abdi, "Partial least square regression (PLS regression)," in Encyclopedia for Research Methods for the Social Sciences, pp. 792-795, SAGE Publications, Inc., California (2003).

30. R. Rosipal and N. Kramer, Subspace, Latent Structure and Feature Selection, pp. 34-51, Springer, New York (2006).

31. T. Mehmood et al., "A review of variable selection methods in partial least squares regression," Chemom. Intell. Lab. Syst. 118, 62-69 (2012).

32. Z. Xu et al., "Back-directional gated spectroscopic imaging for diffuse light suppression in high anisotropic media and its preclinical applications for microvascular imaging," IEEE J. Select. Topics Quantum Electron. 16(4), 815-823 (2010).

33. Z. Xu, A. K. Somani, and Y. L. Kim, "Scattering anisotropy-weighted mesoscopic imaging," J. Biomed. Opt. 17(9), 0905011 (2012).

34. R. L. Konger et al., "Spatiotemporal assessments of dermal hyperemia enable accurate prediction of experimental cutaneous carcinogenesis as well as chemopreventive activity," Cancer Res. 73(1), 150-159 (2013).

35. S.-C. Yoon et al., "Hyperspectral image reconstruction using RGB color for foodborne pathogen detection on agar plates," Proc. SPIE 9024, 90240I (2014).

36. I. Nishidate et al., "Estimation of melanin and hemoglobin using spectral reflectance images reconstructed from a digital RGB image by the wiener estimation method," Sensors 13, 7902-7915 (2013).

37. H.-L. Shen and J. H. Xin, "Spectral characterization of a color scanner by adaptive estimation," J. Opt. Soc. Am. A 21(7), 1125-1130 (2004).

38. K. Barnard and B. Funt, "Camera characterization for color research," Color Res. Appl. 27(3), 152-163 (2002).

39. H. Chang et al., "Whole blood reflectance for assessment of hematologic condition and detection of angiographic contrast media," Appl. Opt. 48(13), 2435-2443 (2009).

40. H. Chang et al., "A novel technique for endovascular detection and removal of radiographic contrast during angiography," J. Cardiol. 21(7), 314-318 (2009).

41. W. C. Vogt et al., "Effects of mechanical indentation on diffuse reflectance spectra, light transmission, and intrinsic optical properties in ex vivo porcine skin," Lasers Surg. Med. 44(4), 303-309 (2012). 
42. L. Lim et al., "Probe pressure effects on human skin diffuse reflectance and fluorescence spectroscopy measurements," J. Biomed. Opt. 16(1), 011012 (2011).

43. S. Ruderman et al., "Analysis of pressure, angle and temporal effects on tissue optical properties from polarization-gated spectroscopic probe measurements," Biomed. Opt. Express 1(2), 489-499 (2010).

44. Y. Ti and W.-C. Lin, "Effects of probe contact pressure on in vivo optical spectroscopy," Opt. Express 16(6), 4250-4262 (2008).

45. R. Reif et al., "Analysis of changes in reflectance measurements on biological tissues subjected to different probe pressures," J. Biomed. Opt. 13(1), 010502 (2008).

46. Y. L. Kim et al., "Simultaneous measurement of angular and spectral properties of light scattering for characterization of tissue microarchitecture and its alteration in early precancer," IEEE J. Select. Topics Quantum Electron. 9(2), 243-256 (2003).

47. I. C. van Wyk, "Anemia in East African short-horn Zebu calves: field diagnosis, infectious causes and pathogen interactions," $\mathrm{PhD}$ thesis, University of Pretoria, Pretoria, South Africa (2012).

48. C. E. Shulman et al., "Screening for severe anemia in pregnancy in Kenya, using pallor examination and self-reported morbidity," Trans. R. Soc. Trop. Med. Hyg. 95, 250-255 (2001).

Taehoon Kim is a PhD student at the Weldon School of Biomedical Engineering, Purdue University, West Lafayette, Indiana.

Seung Ho Choi received his $\mathrm{PhD}$ in biomedical engineering from Purdue University in 2016 . He is currently a postdoctoral fellow at the Weldon School of Biomedical Engineering, Purdue University, West Lafayette, Indiana, USA.

Nathan Lambert-Cheatham received his BS degree in biomedical engineering from Purdue University in 2012 . He is currently a medical student at Maian University, Indianapolis, Indiana, USA.

Zhengbin $\mathrm{Xu}$ received his $\mathrm{PhD}$ in biomedical engineering from Purdue University in 2012 . He is currently a regulatory affairs engineer at Philips Health Care, Cleveland, Ohio, USA.

Janice E. Kritchevsky is a professor of Large Animal Internal Medicine in College of Veterinary Medicine, Purdue University, West Lafayette, Indiana, USA.

Francois-René Bertin is an assistant professor of Equine Internal Medicine, University of Queensland, Gatton, Australia.

Young L. Kim received his $\mathrm{PhD}$ in biomedical engineering and his MS degree in clinical investigation from Northwestern University in 2005 and 2007, respectively. He is currently an associate professor at the Weldon School of Biomedical Engineering and a codirector of Biomedical Sciences Interdisciplinary Graduate Program at Purdue University, West Lafayette, Indiana, USA. His research interests include light-matter (e.g., natural materials and biological tissue) interactions, biophotonics technology development for sensing, imaging, and treatment, and biostatistical analyses. 